\title{
Predictors of Amputation in Patients with Critical Lower Limb Ischemia
}

\author{
Borges Wagner Ramos ${ }^{1}$, Carvalho Aquiles Tadashi Ywata de ${ }^{2}$ and Aras Junior Roque ${ }^{3}$ \\ ${ }^{1}$ Department of Vascular and Endovascular Surgery, Hospital Ana Neri, Federal University of Bahia, Salvador, State of Bahia, Brazil \\ ${ }^{2}$ Department of Vascular and Endovascular Surgery, Hospital Geral Roberto Santos, Salvador, State of Bahia, Brazil \\ ${ }^{3}$ Department of Medicine, Federal University of Bahia, Salvador, State of Bahia, Brazil
}

Corresponding author: Borges Wagner Ramos, Vascular and Endovascular Surgeon, M.S. in Medicine and Health, Department of Vascular and Endovascular, Surgery of the Hospital Ana Neri, Federal University of Bahia, Salvador, State of Bahia, Brazil, Tel:+5589612365; E-mail: wagner2076@bol.com.br

Received date: Oct 10, 2016; Accepted date: Nov 15, 2016; Published date: Nov 23, 2016

Copyright: ( 2016 Ramos WB, et al. This is an open-access article distributed under the terms of the Creative Commons Attribution License, which permits unrestricted use, distribution, and reproduction in any medium, provided the original author and source are credited.

\begin{abstract}
Back Ground: Chronic critical ischemia of the lower limbs with the respective functional loss, continues to be a major public health problem, particularly in developing countries and patients with diabetes. Despite advances in diagnostic methods and innovations in endovascular treatment, amputation is still common, with great psychosocial impact. This study aims to identify possible predictors factors of major amputations in these patients.
\end{abstract}

Materials and Methods: Prospective cohort study was conducted in which selected patients were submitted to clinical and laboratory evaluation, arterial imaging studies and defined study variables.

Results: Between March 2015 and February 2016, 182 patients were selected. Of these, the affected limb was amputated in 66 and salvaged in 116. Amputations were more common among males (40.2\%), smokers (37.2\%), patients with high blood pressure $(40.7 \%)$, and as a result of stroke $(43.5 \%)$, without statistically significant difference. Factors associated with loss of limb were previous amputation $(62 \%, p<0.001)$, trophic lesion $(41.2 \%$, $p<0.018)$, infection $(54.5 \%, p<0.001)$, chronic kidney disease $(67.7 \%, p<0.001)$, elevated creatinine $(3.64 \pm 2.3$, $p<0.001)$, and hemoglobin A1C $(7.79 \pm 0.96 p<0.001)$, with statistical significance. Furthermore, in the group of amputees, the mean ages $(73.5 \pm 12.7, p=0.065)$, hemoglobin $(9.7 \pm 3.8, p=0.689)$, triglycerides $(142.7 \pm 55.4$, $p=0.233)$, and fasting glycemic level $(247 \pm 97, p=0.172)$, were higher, but without statistical significance. The nonamputee group had greater mean cholesterol HDL $(50.9 \pm 8.8, p=0.568)$, cholesterol LDL $(150.9 \pm 35.09 p=0.715)$, ankle-brachial index $(0.14 \pm 0.24 ; p=0.573)$, hospitalization time $(13.9 \pm 11.3, p=0.655)$, follow-up time (11.26 \pm 4.09), and underwent more angioplasties and open revascularizations, but without statistical significance. The rate of limbs salvaged was $63.73 \%$, deaths $9.89 \%$, and follow-up loss $6.04 \%$. The reduced model correctly classifies $90.7 \%$ of the cases with sensitivity of $86.4 \%$ and specificity of $93.1 \%$. In the ROC, the area under the curve was $0.954(p<0.001)$.

Conclusion: Chronic kidney disease, ischemic heart disease, uncontrolled diabetes, arterial aneurysms, previous amputation and lack of preventive care are predictors of risk for major amputation in this population.

Keywords: Amputation; Critical limb ischemia; Diabetic foot

\section{Abbrevations}

OR: Odds Ratio; CI: Confidential Interval; TASC: Trans-atlantic Inter-society Consensus; SD: Standard Deviation P: Probability

\section{Critical Lower Limb Ischemia}

Chronic critical ischemia of the lower limbs with the respective functional loss, continues to be a major public health problem, particularly in developing countries and patients with diabetes. Despite advances in diagnostic methods and innovations in endovascular treatment, amputation is still common, with great psychosocial impact. This study aims to identify possible predictors factors of major amputations in these patients.

Prospective cohort study was conducted in which selected patients were submitted to clinical and laboratory evaluation, arterial imaging studies and defined study variables.
A database was created and analyzed using the Statistical Package for the Social Science software version 21 (SPSS Inc., Chicago, IL, USA). Descriptive analysis was performed using the absolute and relative frequencies for the qualitative variables and the means and standard deviations for the quantitative variables. The KolmogorovSmirnov test (with Lilliefors correction) combined with asymmetry and kurtosis was used to test the normality of data distribution. The level of significance was set at $\mathrm{p}<0.05$ for the rejection of the null hypothesis, i.e., the absence of significant differences in the variables between the groups.

The chi-squared test was used to assess the presence of risk factors for amputation, and simple logistic regression was used to estimate the odds ratio (OR) and 95\% confidence interval (CI). Subsequently, multiple logistic regression was performed, and the final model was constructed using the variables with p-values of $<0.20$ in the crude analysis. After that, the variables with the highest non-significant $\mathrm{p}$ values (Wald test) were removed from the model one at a time. 
In each step of the analysis, the effect of the removal of each variable on the adjustment quality of the model and on its predictive ability was assessed using the Hosmer-Lemeshow test, Nagelkerke R2, and change in the value of the logarithm of the maximum likelihood ratio (-2log). Only four observations were considered outliers, but they remained in the final model because their removal would not affect the significance or quality of the adjustment of the logistic model. The ROC curve and area under the curve (AUC 95\% CI) were calculated to test the discrimination ability of the model.

The study was approved by ANH-FUB research ethics committee under $986548 / 2015$ and all participants signed the consent form.

Between March 2015 and February 2016, 182 patients were selected. Of these, the affected limb was amputated in 66 and salvaged in 116. Amputations were more common among males (40.2\%), smokers (37.2\%), patients with high blood pressure (40.7\%), and as a result of stroke (43.5\%), without statistically significant difference.

Factors associated with loss of limb were previous amputation $(62 \%$, $\mathrm{p}<0.001)$, trophic lesion $(41.2 \%, \mathrm{p}<0.018)$, infection $(54.5 \%, \mathrm{p}<0.001)$, chronic kidney disease $(67.7 \%, \mathrm{p}<0.001)$, elevated creatinine $(3.64 \pm$ 2.3, $\mathrm{p}<0.001)$, and hemoglobin A1C $(7.79 \pm 0.96 \mathrm{p}<0.001)$, with statistical significance. Furthermore, in the group of amputees, the mean ages $(73.5 \pm 12.7, \mathrm{p}=0.065)$, hemoglobin $(9.7 \pm 3.8, \mathrm{p}=0.689)$, triglycerides $(142.7 \pm 55.4, \mathrm{p}=0.233)$, and fasting glycemic level $(247 \pm$ 97, $\mathrm{p}=0.172$ ), were higher, but without statistical significance.

The non-amputee group had greater mean cholesterol HDL (50.9 \pm 8.8, $\mathrm{p}=0.568)$, cholesterol LDL $(150.9 \pm 35.09 \mathrm{p}=0.715)$, ankle-brachial index (0.14 $\pm 0.24 ; p=0.573)$, hospitalization time (13.9 \pm 11.3 , $\mathrm{p}=0.655)$, follow-up time (11.26 \pm 4.09$)$, and underwent more angioplasties and open revascularizations, but without statistical significance. The rate of limbs salvaged was $63.73 \%$, deaths $9.89 \%$, and follow-up loss $6.04 \%$. The reduced model correctly classifies $90.7 \%$ of the cases with sensitivity of $86.4 \%$ and specificity of $93.1 \%$. In the ROC, the area under the curve was $0.954(\mathrm{p}<0.001)$.

Diabetes melito is a disease with growing prevalence and is a major cause of amputations [1,2]. Numerous variables are involved in its etiology and complications. The long duration of the disease and poor metabolic control $[3,4]$ demonstrated by the high glycated hemoglobin and fasting glycemic levels in the amputee groups, reinforce the higher risk of amputation in these patients, as well of the higher frequency of fatal cardiovascular events.

According to the International Consensus on the Diabetic Foot, patients with previous amputation should be followed up every 1-3 months and should have at least one foot examination per year, or more frequently if the risk of complications is high and worsening of the diabetic foot is inevitable through preventative measures and early diagnosis. Lack of access to health services, despite the increased coverage of basic health care in the last decade in Brazil, and low adherence to treatment, despite the development of educational practices in the management for diabetic foot, increased the risk of amputation seen in this population. It is important that they are educated on health and diabetes melito. Public policies which guarantee access to basic medical care (preventive medicine) to make early diagnoses, provide health education and facilitate referral to specialized services are encouraged, where necessary

Various factors are associated with the risk of amputation [5,6]. The profile of severity of the patients studied was multifactorial, and some variables did not remain in the final model of regression logistics. In our population, aortic aneurism and coronary artery disease were predictors of amputation, possibly due to embolization of aneurysms to the distal arterial bed and the already know coronariopaty in the selected cases. A possible explanation is that in the presence of the other, they lose their intensity, e.g., age, infection, trophic lesion and ankle-brachial index. However, they generally behave like risk factors when compared with the others in the population.

Several authors agree that patients with higher mean ages and anemia have a higher risk of amputation, especially in those with diabetes. Anemia is an independent risk factor for amputation and mortality in hospitalized patients with peripheral artery disease [7]. It is emphasized that the amputees group presented higher rates of infection, with some evolving to gangrene and more severe obstructive arterial lesions characterized by TASC C and D, which may have contributed to the outcome of the amputation, even when revascularization was attempted.

It is known that hypervolemia, increased oxidative stress, uremic toxicity, and lipid alterations in the coagulation cascade are included in the genesis of early atherosclerosis of patients with chronic kidney disease. In the studied population, all patients with chronic kidney disease were amputee, despite having been submitted to open revascularization and/or endovascular procedures. The severity of association of risk factors such as arterial calcification, location of the obstruction and/or stenosis (frequent infrapatellar arterial involvement), coronary artery disease, and metabolic state may have contributed to the outcome.

Peripheral artery disease in the distal arterial bed, presents a challenge to vascular and endovascular surgeons. The patency of revascularization procedures in chronic limb ischemia remains a topic of discussion, with a trend, primarily, endovascular treatment [8] due to the shorter hospitalization and surgery times, lower response to surgical trauma, and possibility of treatment of various vessels in a single surgical act, particularly in high-risk patients. However, it is still necessary to consider to use of isolated contrasts, need for a favorable anatomy for access and vascular catheterization, availability of specific materials and rigorous vigilance of the patency of revascularization.

A higher rate of re intervention was observed in angioplasty procedures, even with a promising technique such as the use of stents and pharmacological balloons or multiple angioplasties. This may be justified by the high rates of restenosis in small-caliber arteries, as verified by others authors [9]. Our population showed similar rates of risk for amputation when comparing angioplasty and open revascularization. The durability of endovascular intervention and/or open surgery continues to be greatest challenge in limb salvage [10].

Our data support some predictors of risk for amputation in patients with cronic limb ischemia (previous amputation, coronary artery disease, chronic kidney disease, arterial aneurism, increase in glycated hemoglobin and a lack of previous care), but the participation of other risk factors intrinsic to atherosclerotic disease cannot be ruled out, and the clinical state of these patients that, taken together, are determining factors for poor clinical outcomes, re interventions, cardiovascular events, and limb amputation. Further studies and larger follow-up time are necessary to define the real impact of the variables.

\section{Declaration}

The study was approved by ANH-FUB research ethics committee under $986548 / 2015$ and all participants signed the consent form. 
Citation: $\quad$ Ramos WB, Ywata De TCA, Roque AJ (2016) Predictors of Amputation in Patients with Critical Lower Limb Ischemia. J Diabetes Metab 7: 714. doi:10.4172/2155-6156.1000714

Page 3 of 3

\section{Competing Interests}

The authors have declared that no competing interests exist.

\section{Authors' Contribution}

Wagner Ramos Borges: dates e write / Roque Aras Junior and Aquiles Tadashi Ywata de Carvalho - review.

\section{Acknowledgments}

Ana Néri Hospital - UFBA.

\section{Funding}

This research did not receive any specific grant from funding agencies in the public, commercial, or not-for- profit sectors.

\section{References}

1. International Diabetes Federation (2013) IDF Diabetes Atlas 11: 13-18.

2. Ministry of Health (2002) Secretariat of Health Policies, Department of Strategic Programmatic Actions. Plan to reorganize attention to hypertension and diabetes mellitus: manual hypertension and diabetes mellitus. Ministry of Health 21: 15-20.

3. Konstantitaki V (2008) The role of primary care in the prevention of diabetic foot amputation. Int J Caring Sci 1:26-33.
4. Venermo M, Manderbacka K, Ikonen T, Keskimäki I, Winell K, et al. (2013) Amputations and socioeconomic position among persons with diabetes mellitus, a population-based register study. BMJ Open 3: e 002395.

5. García-Alvarez Y, Lázaro-Martínez, García-Morales E, Cecilia-Matilla A, Aragón-Sánchez J, et al. (2013) Morph functional characteristics of the foot in patients with diabetes mellitus and diabetic neuropathy. Diabetes Metab Syndr 7: 78-82.

6. Yusof NM, Ab Rahman J, Zulkifly AH, Che-Ahmad A, Khalid KA, et al. (2015) Predictors of major lower limb amputation among type II diabetic patients admitted for diabetic foot problems. Singapore Med J 56: 626-631.

7. Luders F, Bunzemeier H, Engelbertz C, Malyar NM, Meyborg M, et al. (2016) CKD and acute and long-term outcome of patients with peripheral artery disease and critical limb ischemia. Clin J Am Soc Nephrol 11: 216-222.

8. Vierthaler L, Callas PW, Goodney PP, Schanzer A, Patel VI, et al. (2015) Vascular Study Group of New England: Determinants of survival and major amputation after peripheral endovascular intervention for critical limb ischemia. J Vasc Surg 62: 655-664.

9. Genovese EA, Chaer RA, Taha AG, Marone LK, Avgerinos E, et al. (2016) Risk Factors for Long-Term Mortality and Amputation after Open and Endovascular Treatment of Acute Limb Ischemia. Ann vasc Surg 30: 82-92.

10. Couto M, Figueróa A, Sotolongo A, Pérez R, Ojeda JM (2015) Endovascular Intervention in the Treatment of Peripheral Artery Disease. Bol Asoc Med P R 107: 47-51. 